\title{
Improvement in Brightness of Multicusp-Plasma Ion Source
}

\author{
$\underline{\text { Q. Ji }}^{\text {a), b) }}{ }^{\text {, X. Jiang }}{ }^{\text {c) }}$, T. -J. King ${ }^{\text {b) }}$, K.-N.Leung ${ }^{\text {c) }}$, K. Standiford ${ }^{\text {d) }}$, and S. B. Wilde \\ Lawrence Berkeley National Laboratory, University of California, Berkeley, CA 94720
}

\begin{abstract}
The beam brightness of a multicusp-plasma ion source has been substantially improved by optimizing the source configuration and extractor geometry. The current density of a $2 \mathrm{keV} \mathrm{He}^{+}$ beam extracted from a 7.5 -cm-diameter source operating at $2.5 \mathrm{~kW}$ RF power is $\sim 100 \mathrm{~mA} / \mathrm{cm}^{2}$, which is $\sim 10$ times larger than that of a beam extracted from a 5 -cm-diameter source operating at $1 \mathrm{~kW}$ RF power. A smaller focused beam spot size is achieved with a counter-bored extractor instead of a conventional ("through-hole") extractor, resulting another order of magnitude improvement in beam current density. Measured brightness can be as high as $440 \mathrm{~A} / \mathrm{cm}^{2} \mathrm{Sr}$, which represents a 30 times improvement over prior work.
\end{abstract}

\section{Introduction}

Maskless ion beam lithography is one of the "next-generation lithography" techniques for integrated-circuit manufacture at the $70 \mathrm{~nm}$ technology node and beyond. The use of ions is

\footnotetext{
a) Electronic mail: qingji@eecs.berkeley.edu

b) also with Department of Electrical Engineering and Computer Sciences, University of California, Berkeley, CA 94720.

c) also with Department of Nuclear Engineering, University of California, Berkeley, CA 94720.

d) also with Keith Standiford Consulting Services, Carmel, CA 93923.
} 
attractive for lithography, because resist sensitivity is almost two orders of magnitude higher for ions than for electrons ${ }^{2}$, and higher resolution is achievable due to reduced proximity effects. A compact focused-ion-beam system incorporating a multicusp-plasma ion source has been under investigation at the Lawrence Berkeley National Laboratory. ${ }^{1,3-5}$ Multicusp-plasma ion sources have been used for many applications, such as neutral-beam injectors for fusion devices, particle accelerators, ion-implantation systems, neutron tubes for oil-well logging, and proton therapy machines. ${ }^{6,7}$ They can produce a uniform plasma over a large area ${ }^{6}$; therefore, a single source can be used to generate a large number of closely packed beamlets for parallel processing $\square$ an advantage which cannot be provided by any other type of ion source.

The brightness of a multicusp-plasma ion source is a key issue for its application to maskless ion beam lithography. If an array of $100 \times 100$ independently controlled beamlets is used to expose a wafer, the brightness for each beamlet at the entrance of the objective lens needs to be $\sim 1000$ $\mathrm{A} / \mathrm{cm}^{2} \mathrm{Sr}$, in order to achieve exposure throughput of sixty $300-\mathrm{mm}$-diameter wafers per hour. ${ }^{1}$ Scott et al. recently reported the measured beam brightness of a 5-cm-diameter multicusp-plasma ion source to be less than $15 \mathrm{~A} / \mathrm{cm}^{2} \mathrm{Sr}$, using 5 - $\mu$ m-diameter extraction apertures, ${ }^{1}$ which falls far short of the brightness requirement.

In this paper, the brightness of a multicusp-plasma ion source has been substantially improved by optimizing the source configuration and extractor geometry. First, a larger source $(7.5 \mathrm{~cm}$ in diameter) is employed to achieve higher source current density. Second, a counter-bored extraction aperture is used to achieve smaller focused beam spot size with much reduced 
aberration. With increased RF power, the brightness of the multicusp-plasma ion source can be sufficiently high to make it suitable for application to high-throughput maskless lithography.

\section{Approaches to Improving Brightness}

The beam brightness $\mathrm{B}$ at a plane is defined as:

$$
B=\frac{I_{\Omega}}{\pi r^{2}}=\frac{I}{\pi r^{2} \cdot \Omega}=\frac{J}{\Omega}
$$

where $I_{\Omega}$ is the angular current density, $r$ is the radius of the beam spot at that plane, $I$ is the total beam current, $\Omega$ is the solid angle of the beam, and $\mathrm{J}$ is the current density at that plane. For a fixed solid angle, higher current density at a given plane results in higher brightness. In this paper, two approaches have been taken to improve the brightness of a multicusp-plasma ion source: increasing the total beam current extracted from the source, and reducing the focused beam spot size.

\section{A. Increasing total beam current}

Figure 1(a) shows the cross-sectional view of a RF-driven multicusp-plasma ion source. An RF discharge is generated using an induction-coil antenna located inside the source. As shown in Figure 1(b), an azimuthal electric field is generated by the time-varying magnetic field inside the antenna loop. Electrons present in the gas volume are accelerated by the induced electric field, and quickly acquire enough kinetic energy to generate a high-density plasma by ionizing the background gas particles. This mode of operation is referred to as the inductively coupled mode, and it is desirable for achieving a high plasma density in the region from which the ion beams are extracted. If the antenna is too close to the wall, strong oscillating electric fields can easily be generated between the antenna and the source chamber, resulting in a capacitively coupled 
discharge in the source wall region. For this reason, it is difficult to operate a 5-cm-diam source with a 3 -cm-diam antenna in the inductively coupled mode, as compared to a 7.5 -cm-diam source with a 4.5 -cm-diam antenna.

Figure 2 compares the extracted $\mathrm{He}^{+}$beam current densities for $7.5-\mathrm{cm}$-diam and 5-cm-diam sources. The current density of the extracted beam increases with RF power for both sources. At $1 \mathrm{~kW}$ of RF input power, corresponding to the source operation condition in Ref. 1, the 7.5$\mathrm{cm}$-source provides $\sim 3$ times higher current density than the 5-cm-source. This difference may be attributed to the different modes of source operation as discussed in the previous section. The current density for the $7.5-\mathrm{cm}$-diam source is over $100 \mathrm{~mA} / \mathrm{cm}^{2}$ at $2.5 \mathrm{~kW}$ of RF power, which is $\sim 10$ times larger than that of the 5 -cm-diam source operating at $1 \mathrm{~kW}$ of $\mathrm{RF}$ power.

\section{B. Reducing focused beam spot size}

The effect of the two different extractor geometries on beam aberrations has been investigated: "through-hole" and counter-bored hole. As shown in Figure 3, the through-hole consists of a uniform-diameter $(50 \mu \mathrm{m})$ aperture, $250 \mu \mathrm{m}$ deep; the diameter of the counter-bored hole changes from $50 \mu \mathrm{m}$ (for the first $250 \mu \mathrm{m}$ of depth) to $300 \mu \mathrm{m}$ (for the second $250 \mu \mathrm{m}$ of depth) facing downstream.

Simulations were performed using the Munro $\operatorname{code}^{8}$ and the results are summarized in Table 1. The aberration for the through-hole aperture is much larger than the focused Gaussian beam spot size. For an image $1 \mathrm{~mm}$ away from the extraction aperture, both geometries provide $\sim 4$ times reduction in image size. The through-hole generates almost one order of magnitude higher aberrations (spherical, coma, field curvature, astigmatism, and distortion) than the counter-bored 
hole. Thus, the counter-bored-hole geometry can substantially reduce the beam aberrations and hence the focused beam spot size.

A simple exposure experiment was performed to verify the simulation results (Figure 3). A 2 $\mathrm{keV} \mathrm{Ar}^{+}$beam generated by a multicusp ion source operating at $500 \mathrm{~W}$ RF power was used to sputter a thin metal foil coated with dry erase ink for about $90 \mathrm{~s}$. The target was located at $1 \mathrm{~mm}$ away from the extraction aperture. With a through-hole extraction aperture, a feature size of 56 $\mu \mathrm{m}$ was obtained. In contrast, a feature size of $12.5 \mu \mathrm{m}$ was achieved with a counter-bored aperture. These results agree with the simulation results very well. With a counter-bored extractor, the focused beam spot size is about four times smaller, yielding another factor of ten increase in focused beam current density.

\section{Brightness Measurement}

\section{A. Experimental setup}

The setup for the brightness measurement is shown in Figure 4. A helium plasma, generated by a 7.5 -cm-diameter source, was used to illuminate a $50-\mu \mathrm{m}$-diameter counter-bored extraction aperture, which was at the same potential as the source chamber. The same brightness analyzer used in Ref. 1 was positioned $1 \mathrm{~mm}$ away from the extraction apertures to measure the beam profile. By scanning the voltages applied to deflector plates, the beam was swept over a $100 \mu \mathrm{m}$ slit located beyond the electrodes. Any current passing the slit was measured by a Faraday cup.

The experimental setup was the same as for the prior work in Ref. 1, except for the following: 
1. A 7.5-cm-diameter source was used instead of a 5-cm-diameter source. As mentioned above, the larger source is more easily operated in the inductively coupled mode, which provides a higher plasma density.

2. A $50 \mu \mathrm{m}$ counter-bored aperture was used to extract the ion beam instead of a $5 \mu \mathrm{m}$ through-hole aperture. The focused beam spot size is smaller, due to reduced aberrations.

3. The beam energy was $2 \mathrm{keV}$ instead of $3 \mathrm{keV}$. No bias voltage was applied between the source and the extractor.

4. The RF and extraction high-voltage power supplies were pulsed at a duty cycle of $2 \%$, to prevent the secondary $5 \mu \mathrm{m}$ aperture from being damaged by the beam.

\section{B. Results and Discussion}

The beam profiles at the $100 \mu \mathrm{m}$ slit for RF power of 1.0, 1.5, and $2.5 \mathrm{~kW}$ are shown in Figure 5. The beam semi-angles are all $\sim 27 \mathrm{mrad}$ for the different power levels. The total beam current passing the secondary $5 \mu \mathrm{m}$ aperture can be calculated by integrating the beam profile. Thus, the current density at the secondary aperture can be easily obtained. The beam brightness was determined using Eqn. (1), and the results are shown in Figure 6. It can be seen that the beam brightness increases with RF power, reaching $440 \mathrm{~A} / \mathrm{cm}^{2} \mathrm{Sr}$ at $2.5 \mathrm{~kW}$. Compared to the previous results ${ }^{1}$, a factor of 30 improvement in brightness is achieved.

\section{SUMMARY}

The brightness of a multicusp ion source can be high if it is operated in the inductively coupled mode and if the beam extraction system is optimized to reduce aberrations. For a $7.5-\mathrm{cm}-$ diameter source with a $50-\mu \mathrm{m}$ counter-bored extraction aperture, the measured brightness (440 
$\mathrm{A} / \mathrm{cm}^{2} \mathrm{Sr}$ ) is significantly higher than in previously reported work. Additional increases in RF power as well as improved source design are being investigated to further improve the brightness in order to meet the requirement of $1000 \mathrm{~A} / \mathrm{cm}^{2} \mathrm{Sr}$ for high-throughput maskless lithography.

\section{ACKNOWLEDGMENTS}

This work is supported by DARPA and the U.S. Department of Energy under contract No. DEAC03-76SF0098. The authors would like to thank T. McVeigh, P. Wong, and J. Fischer for electrical and mechanical support.

\section{REFERENCES}

${ }^{1}$ K.L.Scott, T.-J.King, K.-N.Leung, and R. F. Pease, J. Vac. Sci. Technol. B 19, 2602 (2001).

2 J. L. Bartelt, C. W. Slayman, J. E. Wood, J. Y. Chen, C. M. McKenna, C. P. Minning, J. F.

Coakley, R. E. Holman, and C. M. Perrygo, , J. Vac. Sci. Technol. 19, 1166 (1981).

${ }^{3}$ Q. Ji, T.-J. King, Y.Y. Lee, K.-N. Leung, Proceedings of the SPIE - The International Society of Optical Engineering, 3777, 175(1999).

${ }^{4}$ Q. Ji, T.-J. King, K.-N. Leung, and S. B. Wilde, Rev. Sci. Instrum. 73, 822 (2002).

${ }^{5}$ J. Reijonen, Q. Ji, T.-J. King, K.-N.Leung, A. Persaud, and S. B. Wilde, J. Vac. Sci. Technol. B 20, 180 (2002).

${ }^{6}$ K.N. Leung, J. Vac. Sci. Technol. B 17, 2776 (1999).

${ }^{7}$ K.N. Leung, Rev. Sci. Instrum. 69, 998 (1998).

${ }^{8}$ Provided by MEBS Ltd., 14 Cornwall Gardens, London SW7 4AN, England. 


\section{Figure and Table Captions:}

Figure 1. (a) Cross-sectional view of a RF-driven multicusp-plasma ion source. (b) Top view of the source. Two modes of plasma generation: capacitively coupled and inductively coupled.

Figure 2. Comparison of extracted beam current densities for different size multicusp-plasma ion sources.

Figure 3. Comparison of exposure results using (a) counter-bored hole and (b) through hole as extraction aperture. $2 \mathrm{keV} \mathrm{Ar}^{+}$beams generated by a multicusp source at $500 \mathrm{~W}$ RF power were used to sputter a thin metal foil for about $90 \mathrm{sec}$. The target was located $1 \mathrm{~mm}$ away from the extraction aperture.

Figure 4. Experimental set-up for brightness measurement.

Figure 5. Beam profile at the $100 \mu \mathrm{m}$ slit, for 1.0, 1.5, and $2.5 \mathrm{~kW}$ RF power.

Figure 6. Brightness of $2 \mathrm{keV} \mathrm{He}{ }^{+}$beams as a function of $\mathrm{RF}$ power.

Table 1. Comparison of simulation results for a $50 \mu \mathrm{m}$ through-hole and counter-bored extraction aperture. The image is $1 \mathrm{~mm}$ away from the extraction aperture. 


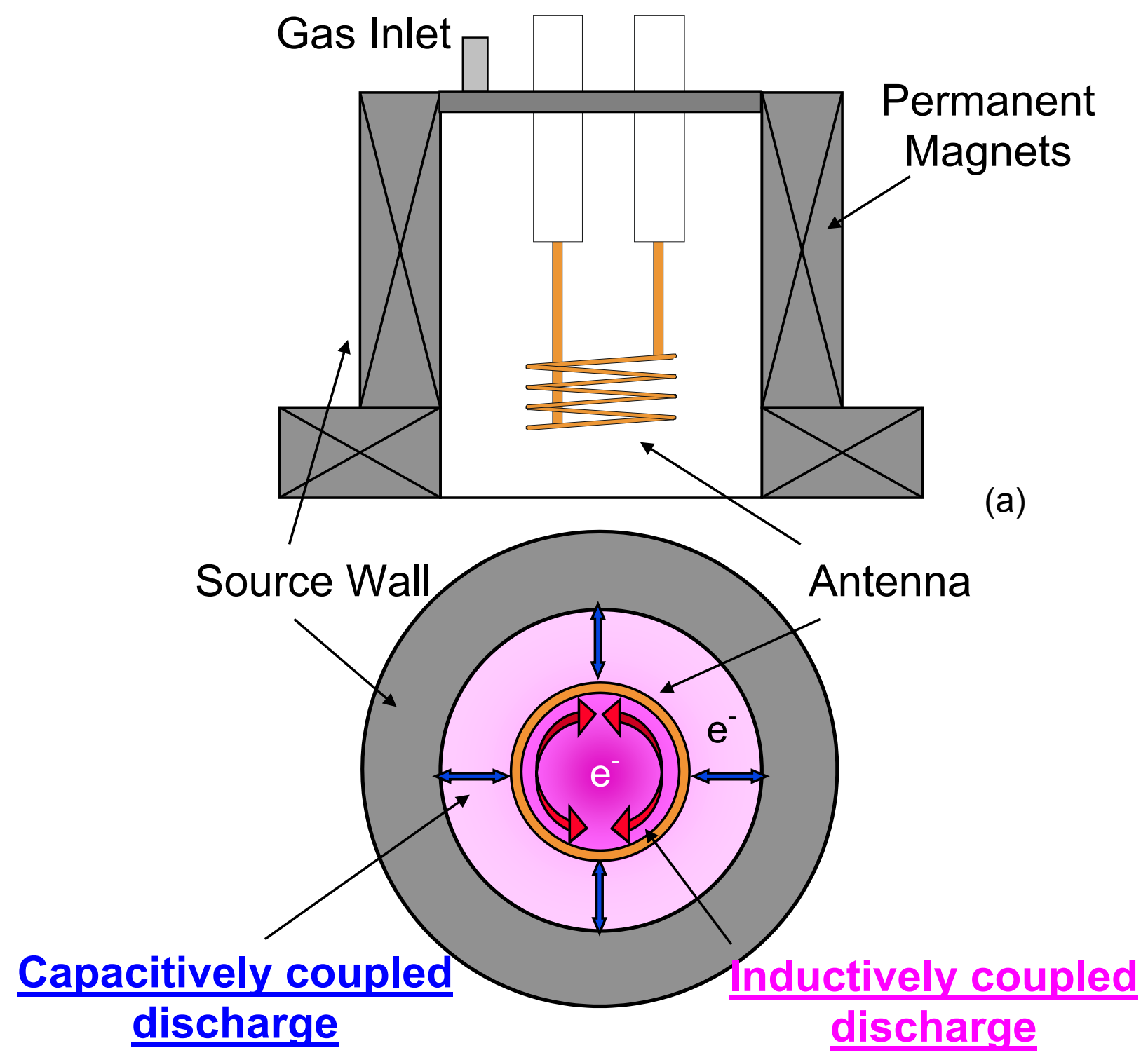

(b)

Figure 1 


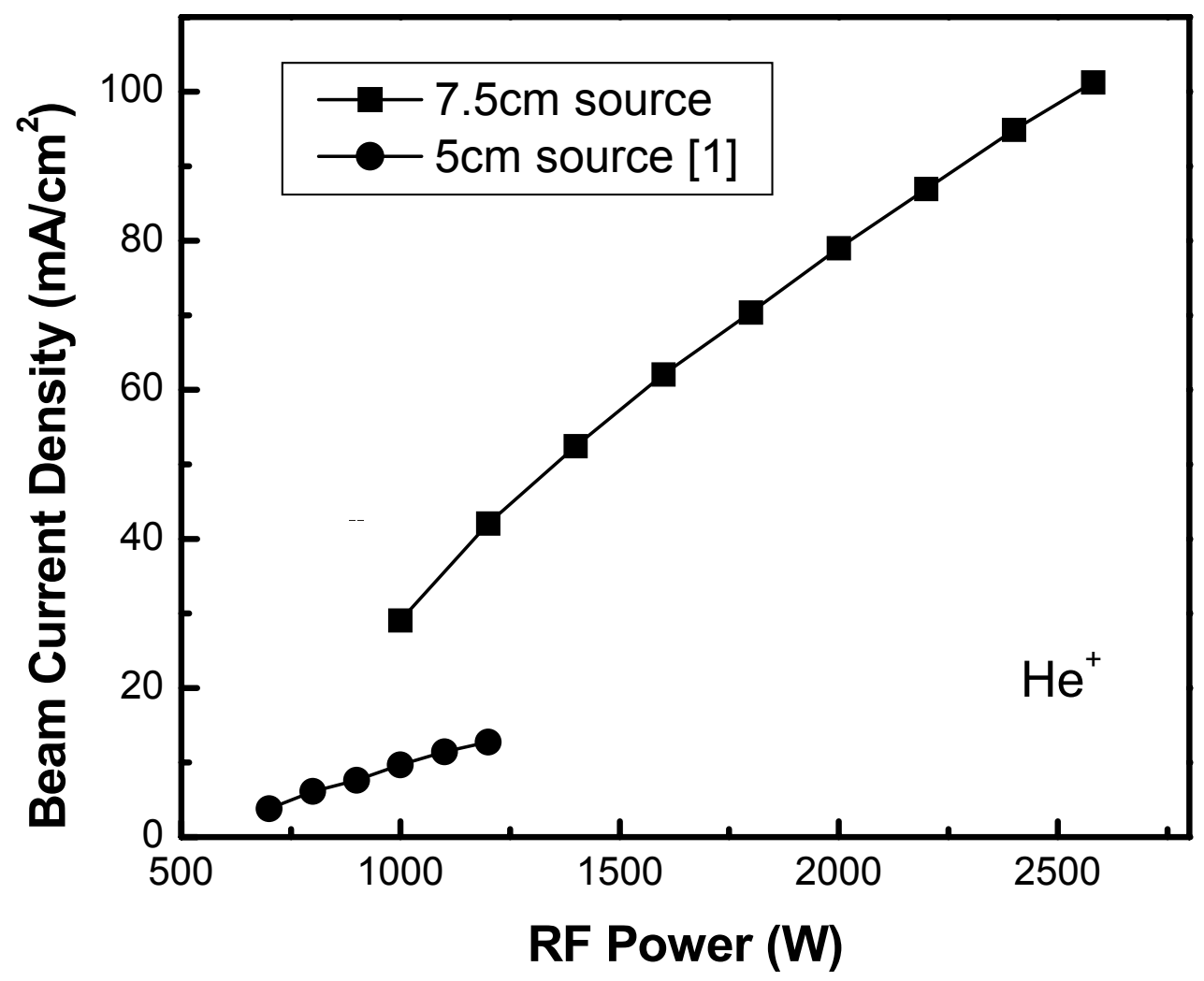

Figure 2 


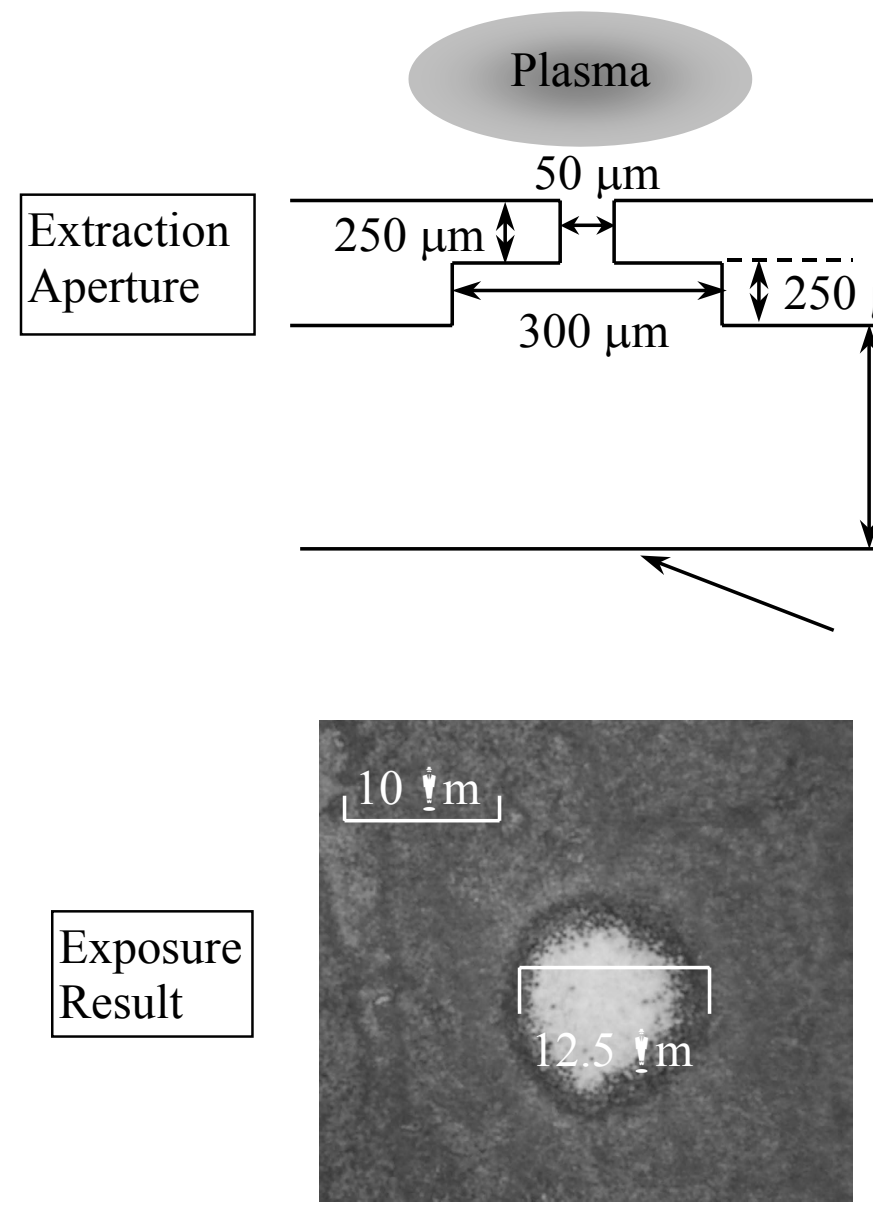

(a)

\section{Plasma}

$50 \mu \mathrm{m}$
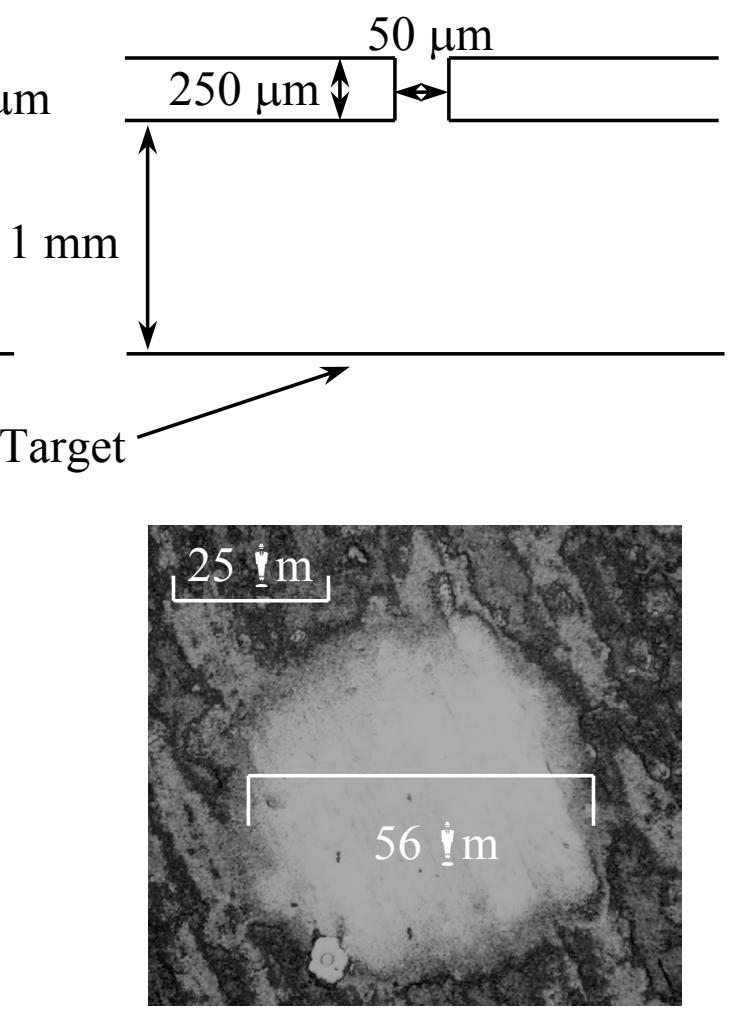

(b)

Figure 3 


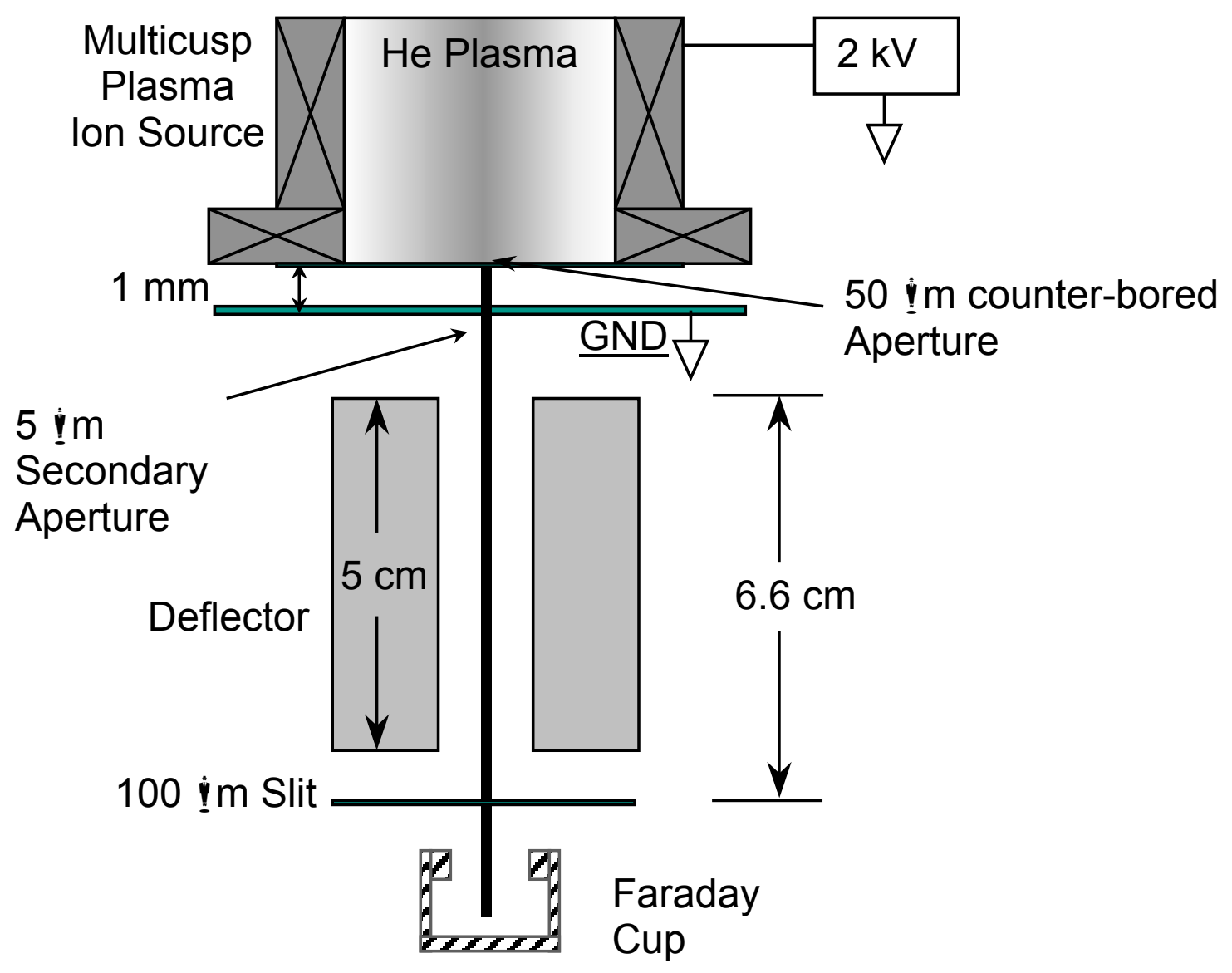

Figure 4 


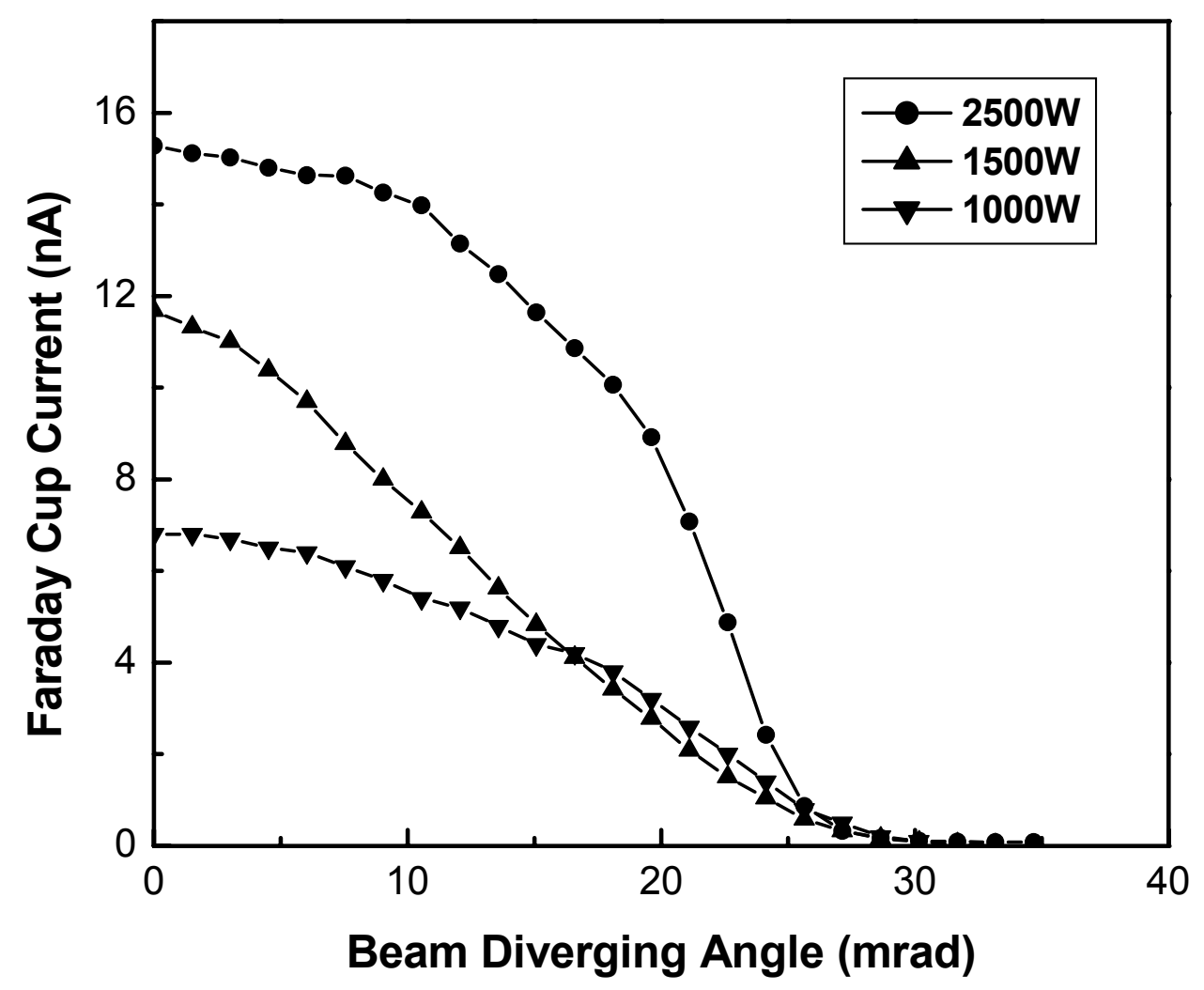

Figure 5 


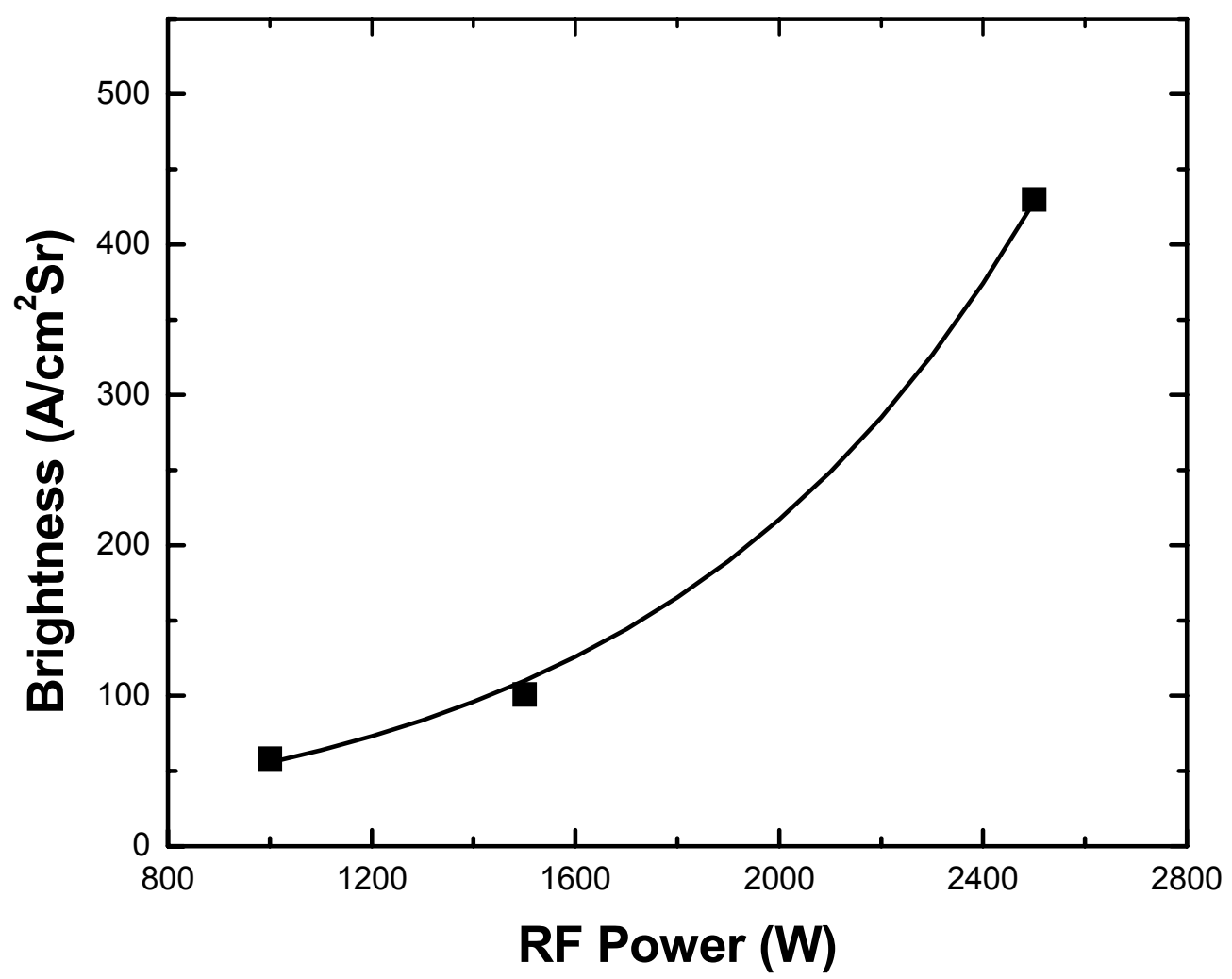

Figure 6 
Table 1

\begin{tabular}{|c|c|c|c|c|c|c|c|c|}
\hline Extraction & Beam & Total \\
geometry & $\begin{array}{c}\text { Size } \\
\text { blur } \\
(\mu \mathrm{m})\end{array}$ & $(\mu \mathrm{m})$ & Spherical & Coma & $\begin{array}{c}\text { Field } \\
\text { curvature }\end{array}$ & Astigmatism & Distortion & Chromatic \\
\hline $\begin{array}{c}\text { Through } \\
\text { hole }\end{array}$ & 16.4 & 46.4 & 14.3 & 31.4 & 27.6 & 16.8 & 3.39 & 2.19 \\
\hline $\begin{array}{c}\text { Counter- } \\
\text { bored } \\
\text { hole }\end{array}$ & 13.1 & 4.51 & 1.58 & 2.99 & 2.31 & 1.13 & 0.24 & 1.49 \\
\hline
\end{tabular}

